\title{
A Randomized Trial for the Treatment of Refractory Status Epilepticus
}

\author{
Andrea O. Rossetti - Tracey A. Milligan • \\ Serge Vulliémoz $\cdot$ Costas Michaelides $\cdot$ \\ Manuel Bertschi $\cdot$ Jong Woo Lee
}

Published online: 28 September 2010

(C) Springer Science+Business Media, LLC 2010

\begin{abstract}
Background Refractory status epilepticus (RSE) has a mortality of 16-39\%; coma induction is advocated for its management, but no comparative study has been performed. We aimed to assess the effectiveness (RSE control, adverse events) of the first course of propofol versus barbiturates in the treatment of RSE.

Methods In this randomized, single blind, multi-center trial studying adults with RSE not due to cerebral anoxia, medications were titrated toward EEG burst-suppression for $36-48 \mathrm{~h}$ and then progressively weaned. The primary endpoint was the proportion of patients with RSE controlled after a first course of study medication; secondary endpoints included tolerability measures.
\end{abstract}

This study is dedicated to the memory of Edward B. Bromfield, MD (1951-2009), who greatly contributed to the study design, and was PI of the American side of the study until his death.

A. O. Rossetti $(\square)$

Department of Neurology, CHUV et Université de Lausanne,

CHUV-BH07, CH-1011 Lausanne, Switzerland

e-mail: andrea.rossetti@chuv.ch

T. A. Milligan - J. W. Lee

Department of Neurology, Brigham and Women's Hospital,

Harvard School of Medicine, Boston, MA, USA

S. Vulliémoz

Department of Neurology, HUG, Geneva, Switzerland

C. Michaelides

Department of Neurology, Massachusetts General Hospital,

Harvard School of Medicine, Boston, MA, USA

M. Bertschi

Department of Neurology, Inselspital, Berne, Switzerland
Results The trial was terminated after 3 years, with only 24 patients recruited of the 150 needed; 14 subjects received propofol, 9 barbiturates. The primary endpoint was reached in $43 \%$ in the propofol versus $22 \%$ in the barbiturates arm $(P=0.40)$. Mortality (43 vs. $34 \%$; $P=1.00)$ and return to baseline clinical conditions at 3 months (36 vs. $44 \%$; $P=1.00$ ) were similar. While infections and arterial hypotension did not differ between groups, barbiturate use was associated with a significantly longer mechanical ventilation $(P=0.03)$. A non-fatal propofol infusion syndrome was detected in one patient, while one subject died of bowel ischemia after barbiturates. Discussion Although undersampled, this trial shows significantly longer mechanical ventilation with barbiturates and the occurrence of severe treatment-related complications in both arms. We describe practical issues necessary for the success of future studies needed to improve the current unsatisfactory state of evidence.

Keywords Propofol - Thiopental - Pentobarbital . Burst-suppression · Outcome $\cdot$ Complications

\section{Introduction}

Status epilepticus (SE) resistant to one-first-line (benzodiazepines, BDZ) and one-second-line (phenytoin, phenobarbital, or valproic acid) antiepileptic drug (AED) is labeled as refractory status epilepticus (RSE) [1]. It develops in $23-44 \%$ of SE patients and has a mortality of 16-39\% [1-4]. Since SE tends to become more resistant to conventional treatment with time and the number of AED used [5], coma induction with barbiturates, propofol (PRO), or midazolam is advocated after failure of second-line treatments $[6,7]$. 
Most of the existing studies on RSE deal with case series of patients receiving a single coma-inducing AED. A metaanalysis did not disclose any significant difference of shortterm mortality among barbiturates (such as thiopental (THP) in use in Europe, or its metabolite pentobarbital (PTB) in North America), PRO, and midazolam; however, treatment allocation was not randomized, patients receiving barbiturates tended to be treated more often under EEG monitoring and experienced more often significant arterial hypotension, and an unclear number of anoxic subjects were included [8]. Furthermore, a single-center retrospective survey of RSE treatment taking into account several strategies (drugs combinations, monotherapy) did not show any outcome difference [4]. Despite the clinical impact of RSE and the call of several authorities for a controlled comparative study [9-11], no attempt has been undertaken so far, possibly also due to the lack of a consensus regarding the optimal therapeutic protocol $[12,13]$.

This prospective study was undertaken to assess the effectiveness (SE control, adverse events) of a first course of PRO versus barbiturates, the two most commonly used agents according to the aforementioned surveys.

\section{Methods}

This was a pragmatic, randomized, single blind, multicenter clinical trial (see Appendix) comparing PRO with barbiturates for RSE treatment. Markedly different physical appearance, pharmacokinetics, and side effects profiles of the drugs represent major difficulties for a double blind study. THP was used in Switzerland, and PTB in the USA; the pharmacological equivalence of THP and PTB was independently confirmed for the aim of this study (E. Perucca, Pavia, Italy, personal communication, August 2005). The study was approved by the IRB of each participating institution.

\section{Patients}

We included adults (older than 16 years) with RSE not due to cerebral anoxia, defined as ongoing clinical or electrographical seizures, or repetitive seizures without return to the baseline for at least $30 \mathrm{~min}$ despite administration of at least one-first-line (benzodiazepine), and one-second-line AED (mostly phenytoin, valproate, phenobarbital, and levetiracetam) in adequate doses, who were clinically determined to require coma induction. Patients with known pregnancy, known intolerance to the study drugs, mitochondrial disorders, egg allergy, hypertriglycerydemia ( $>5 \mathrm{mmol} / \mathrm{l})$, or significant rhabdomyolysis $(\mathrm{CK}>1500 \mathrm{U} / \mathrm{l})$ on admission were excluded.
Baseline assessment included demographics, a simplified functional score before the SE $(0=$ completely independent; 1 = partially dependent; 2 = completely dependent), history of previous seizures, concurrent medications, time to first SE treatment administration, seizure type (simple partial, complex-partial, generalized convulsive, nonconvulsive status in coma), consciousness before first SE treatment, blood tests (creatinine, ASAT, ALAT, $\gamma \mathrm{GT}$, triglycerides, CK, CRP), serum AED levels, and an urine pregnancy test in women aged 18-50. The STESS (status epilepticus severity score, [14]) was calculated subsequently using age, previous seizure history, consciousness, and seizure type.

\section{Intervention}

After written consent was obtained by proxy, randomization (PRO: barbiturates; 1:1) was stratified by institution, using blocks with sealed envelopes. Study drugs were administered as follows. PTB (USA): bolus of $5 \mathrm{mg} / \mathrm{kg} \mathrm{IV}$, then titration toward burst-suppression or, if no EEG available, toward $2 \mathrm{mg} / \mathrm{kg} / \mathrm{h}$ until EEG was available. THP (Switzerland): bolus of $2 \mathrm{mg} / \mathrm{kg} \mathrm{IV}$, then titration toward burst-suppression or, if no EEG available, toward $4 \mathrm{mg} / \mathrm{kg}$ / h until EEG was available. PRO (USA and Switzerland): bolus of $2 \mathrm{mg} / \mathrm{kg}$, then titration toward burst-suppression or, if no EEG available, toward $5 \mathrm{mg} / \mathrm{kg} / \mathrm{h}$ until EEG was available. In each arm, a BDZ was administered at low dose (lorazepam: $4 \mathrm{mg} / 24 \mathrm{~h}$, or clonazepam $2 \mathrm{mg} / 24 \mathrm{~h}$ ) throughout the study period, to reduce required doses of study drugs [15]. The second-line $\operatorname{AED}(\mathrm{s})$ were administered in usual daily doses. Interruption criteria were a PRO infusion syndrome (PRIS) with any of the following: $\mathrm{CK}>2000 \mathrm{U} / \mathrm{l}$, triglycerides $>5.3 \mathrm{mmol} / \mathrm{l}$ (=500 mg/ $\mathrm{dl}$ ), or progressive lactic acidosis $>6 \mathrm{~h}$ after treatment initiation $(>2.5 \mathrm{mmol} / \mathrm{l}$ with bicarbonate $<20 \mathrm{mmol} / \mathrm{l})$ [16], not due to sepsis, and after lactate normalization. Furthermore, treatment-refractory arterial hypotension (as judged necessary to reverse by the attending physician) and hypersensitivity reaction with rash, fever, and lymphadenopathy also represented interruption criteria, implying discontinuation of the study drug.

EEG monitoring had to be started at least within $12 \mathrm{~h}$ of the beginning of the intervention. Burst-suppression (5-15 s interburst intervals) was continued for 36-48 h under EEG control, after which the study drug was progressively weaned over at least $24 \mathrm{~h}$. The treating clinician, if considering that RSE remained insufficiently controlled, according to the pragmatic intent of the study was allowed to pursue the best treatment (retry the study drug, or switch it, or combine it with another compound). The controlled application of the study drug ended after the first weaning attempt. 
Daily assessment (during the controlled study drug application, and until $48 \mathrm{~h}$ after evaluation of treatment success) included clinical and EEG seizure activity, central body temperature, determination of triglycerides, CK, CRP, glucose, blood gas analysis, lactate (at least twice a day), blood levels and dosage of other administered AED, episodes of oxygen desaturation $<90 \%$, episodes of arterial hypotension (systolic $\mathrm{BP}<90 \mathrm{mmHg}$ refractory to volume expansion and requiring vasopressors), cardiac arrhythmia (sustained supraventricular or ventricular tachycardia, bradycardia $<50 \mathrm{bpm}$, new heart conduction blocks) associated with hemodynamic instability and requiring treatment, infections requiring antibiotic treatment, doppler/duplex confirmed deep vein thrombosis, scintigraphy or CT confirmed pulmonary embolism, and administered dose of the study drug.

Long-term assessment (3 weeks and 3 months) was performed using hospital charts and semi-structured phone interviews, and included SE etiology (classified according to [17]) and the simplified functional score ( $0-3$, as above; 3 = death).

\section{Statistical Analysis}

The primary objective was to compare the proportion of patients with successful RSE control after 36-48 h of a stable burst-suppression pattern, defined by all the following criteria: patient alive; no clinical need to re-titrate the study drug to a higher dose during the first 7 days after initial weaning of the study drug; and $\leq 1$ discrete seizure (defined electrographically as evolving focal or generalized pattern for more than $5 \mathrm{~s}$ and less than $2 \mathrm{~min}$ ) per hour during the $2 \mathrm{~h}$ after which the EEG became continuous (a single seizure lasting more than 2 min was sufficient for a treatment failure). In case of PLEDs (periodic lateralized epileptiform discharges), the average interdischarge interval had to be $>1$ s. Persistent interictal epileptiform EEG activity was not counted as treatment failure. A total of 150 patients, 75 in each treatment arm, would be needed to detect an absolute difference of $22 \%$ (relative difference $=30 \%$ ) of the primary outcome toward the worse treatment, with $\alpha=0.05$ (two-sided) and $\beta=0.2$. It was assumed that 13 centers recruiting each $4-5$ patients per year over 3 years would be needed.

Secondary outcome measures were: the functional clinical outcome at day 21 , and at 3 months according to the functional score, length of ventilator treatment in survivors, incidence of thromboembolism, incidence of infectious complications, incidence of hypotension requiring specific treatment, and incidence of PRIS or other severe complications.

Calculations were performed using the release 9 of the Stata software (College Station, TX), applying 2-sided Fisher exact tests for comparisons of categorical data, and Mann-Whitney $U$ tests for continuous data. All the outcomes were analyzed per intention to treat in patients having received at least one dose of the study drug.

\section{Results}

Between November 2006 and December 2009, 24 patients were randomized in five centers (see Appendix, Fig. 1), while five other centers did not recruit any subject and
Fig. 1 Flow diagram of the study

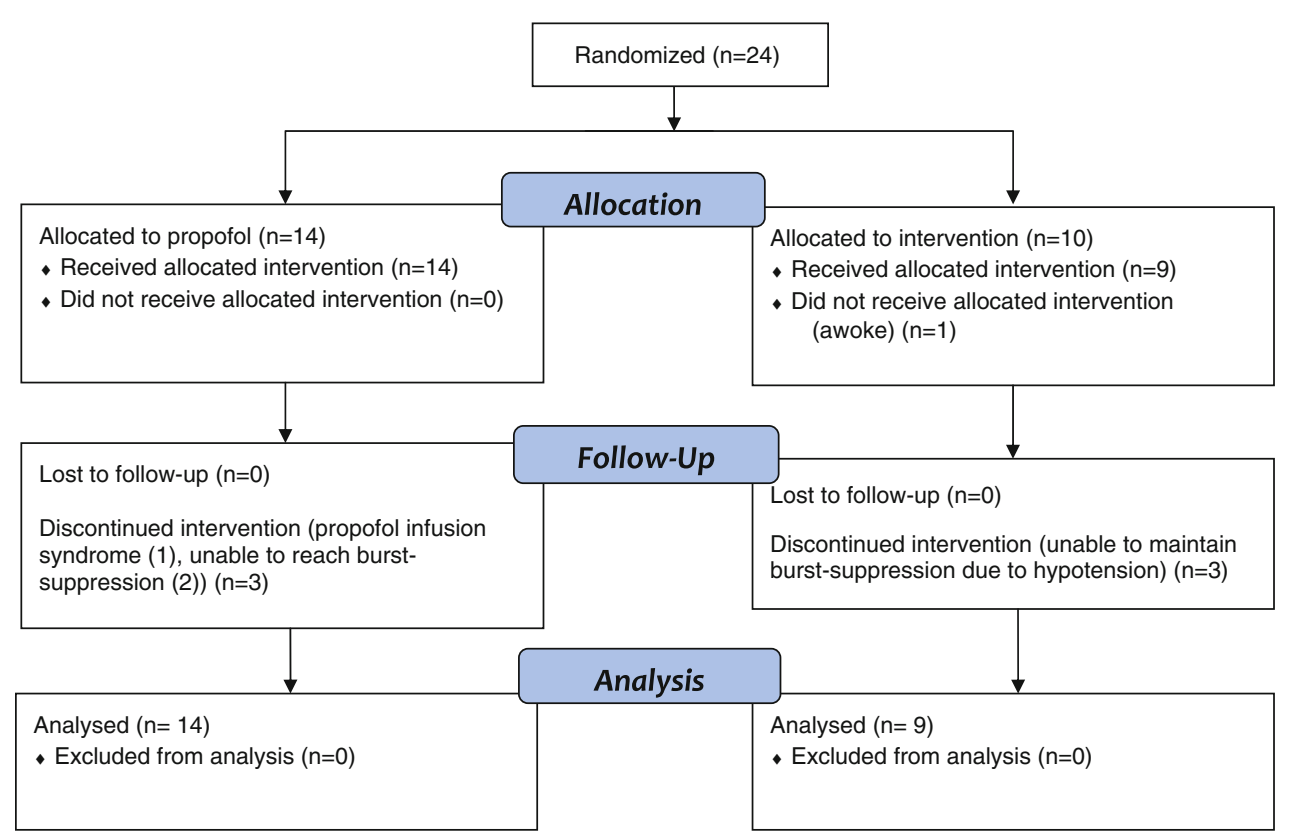


Table 1 Baseline patients' characteristics according to administered treatment

\begin{tabular}{lllll}
\hline & Propofol & BBT & $P$ & Test \\
\hline Patients & 14 & 9 & & \\
Female gender & $7(50 \%)$ & $6(66 \%)$ & 0.67 & Fisher \\
Age (years, median, range) & $57(26-87)$ & $64(16-78)$ & 0.95 & $U$ \\
Acute symptomatic RSE & $8(57 \%)$ & $7(77 \%)$ & 0.34 & Fisher \\
Previous epilepsy & $6(43 \%)$ & $6(66 \%)$ & 0.40 & Fisher \\
Simple- or Complex- & $5(36 \%)$ & $5(55 \%)$ & 0.42 & Fisher \\
$\quad$ partial SE & & & & \\
STESS $\geq 3$ & $8(57 \%)$ & $3(33 \%)$ & 0.40 & Fisher \\
SE treatment initiated $>1 \mathrm{~h}$ & $9(64 \%)$ & $7(77 \%)$ & 0.66 & Fisher \\
\hline
\end{tabular}

$B B T$ barbiturates; $R S E$ refractory status epilepticus; $S E$ status epilepticus; STESS status epilepticus severity score, where $\geq 3$ is considered severe [14]

three withdrew their participation. At the leading recruiting center (CHUV Lausanne), only 10/18 (55\%) of the eligible patients were recruited (mainly because the on-call neurologist did not activate the protocol); this proportion may be even lower in other hospitals. Owing to this insufficient recruitment, the study was terminated.

One patient did not receive the allocated treatment (THP) because he started to awaken shortly after randomization. We present here the results of 23 patients who received at least one study drug dose. Table 1 summarizes baseline characteristics, including SE episode severity, which did not differ significantly among the groups. Etiologies were very variable: in the PRO versus barbiturate groups, there were 3 versus 1 cryptogenic causes, 2 versus 2 acute infections, 3 versus 0 acute strokes, 1 versus 2 AED withdrawals, 1 versus 0 de novo CJD, and 0 versus 1 autoimmune encephalitis, 0 versus 1 severe metabolic disturbances, 2 versus 2 ancient strokes or brain traumas, and 2 versus 1 brain tumors.

As seen in Fig. 1, in the PRO, arm treatment was interrupted prematurely in three patients: one had a PRIS (see below); the others could not reach a satisfactory burstsuppression pattern under infusions speed in agreement with hospital's guidelines (discouraging in the US participating Centers PRO administration at rates higher than $5 \mathrm{mg} / \mathrm{kg} / \mathrm{h}$ ). Interruptions occurred also on the barbiturates arm (three subjects), all following treatment-refractory hypotension preventing adequate dosage to reach a burstsuppression pattern. The outcome measures are illustrated in Table 2; the primary outcome was reached in $43 \%$ patients treated with PRO and $22 \%$ with barbiturates, and the majority of the patients who failed the first RSE treatment course were subsequently controlled. Functional outcome at 3 weeks and 3 months, as well as in-hospital mortality, was comparable among the treatment arms.

Regarding side effects, there was no meaningful difference among the groups regarding thromboembolic, infectious, or hypotensive complications. In one patient, PRO was interrupted within the first $24 \mathrm{~h}$ because of a beginning PRIS (lactate rise from 1.1 to $2.5 \mathrm{~mm} / \mathrm{l}$, braycardia to $28 \mathrm{bpm}$; PRO infusion rate $5.5 \mathrm{mg} / \mathrm{kg} / \mathrm{h}$ at that time). She was treated with midazolam and left the hospital alive (but did not return to baseline at 3 months, likely due to the underlying encephalitis). In another subject who

Table 2 Outcome data

\begin{tabular}{llll}
\hline & Propofol & BBT & $P$ \\
\hline Patients & 14 & 9 & Test \\
Efficacy & & & \\
RSE controlled with first course of study drug & $6(43 \%)$ & $2(22 \%)$ & 0.40 \\
RSE treated subsequently & $4 / 8(50 \%)$ & $5 / 7(71 \%)$ & 1.00 \\
Functional outcome at 3 weeks (returned to baseline) & $5(36 \%)$ & $3(33 \%)$ & 1.00 \\
Functional outcome at 3 months (returned to baseline) & $5(36 \%)$ & $4(44 \%)$ & 1.00 \\
Mortality & $6(43 \%)$ & $3(33 \%)$ & 1.00 \\
Tolerability & & & Fisher \\
Thrombotic/embolic complication & 0 & 0 & 1.00 \\
Infections requiring antibiotics & $7(50 \%)$ & $6(66 \%)$ & 0.67 \\
Hypotension requiring specific treatment & $7(50 \%)$ & $5(55 \%)$ & 1.00 \\
Other severe complications & $1(7 \%)^{\mathrm{a}}$ & $1(11 \%)^{\mathrm{b}}$ & Fisher \\
Study drug administration (days, median, range) & $2.5(0-7)$ & $2(0-4)$ & 1.00 \\
Intubation time in survivors (days, median, range) & $4(2-28)$ & $13.5(8-70)$ & 0.45
\end{tabular}

$B B T$ barbiturates; $R S E$ refractory status epilepticus

${ }^{\text {a }}$ Propofol infusion syndrome (PRIS), not fatal

b Ileus with diffuse intestinal ischemia, fatal 
received THP, massive intestinal ischemia developed shortly after THP weaning (EEG still suppressed), and the patient died; he has been previously described in detail [18].

Maximal PRO infusion rates were similar in Switzerland (nine patients; median $5.5 \mathrm{mg} / \mathrm{kg} / \mathrm{h}$, range 2-10.9) and the US (five patients; median $5 \mathrm{mg} / \mathrm{kg} / \mathrm{h}$, range 2-7.1). In the barbiturates arm, THP was used in seven patients (in Switzerland; median $7 \mathrm{mg} / \mathrm{kg} / \mathrm{h}$, range 4-20) and PTB in two subjects (in the US; 2 and $3 \mathrm{mg} / \mathrm{kg} / \mathrm{h}$ ). Excluding subjects in whom the study agent was interrupted, a burstsuppression pattern was achieved under a median of $5 \mathrm{mg}$ / $\mathrm{kg} / \mathrm{h}$ (range 2-10.9) for PRO and $6.7 \mathrm{mg} / \mathrm{kg} / \mathrm{h}$ (range 4-20) for THP. Dose weanings occurred over $48 \mathrm{~h}$ in all patients apart from two subjects on PRO (96 h). Despite the fact that PRO was administered somewhat longer than barbiturates, the only statistically significant difference was related to the longer mechanical ventilation in patients treated with barbiturates. During RSE treatment, several AEDs were prescribed concomitantly, without differences among the groups (phenytoin, levetiracetam, topiramate, and valproate more often than lamotrigine, phenobarbital, and pregabalin).

\section{Conclusion}

This trial had to be prematurely stopped because of insufficient recruitment; however, to the best of our knowledge it represents the first attempt to prospectively compare PRO and barbiturates for RSE treatment, and carries three important messages. First, while RSE control seemed somewhat more frequent (albeit not significantly) after PRO, medium-term functional outcome and mortality appear similar in the two groups. Second, it demonstrates in a randomized trial that the duration of mechanical ventilation was significantly longer in patients treated with barbiturates. Third, such a study was difficult to carry out because of several major issues that need to be addressed in any future attempts.

This study did not disclose any significant difference between PRO and barbiturates in terms of RSE control, mortality, or functional outcome. Premature interruption of study drug administration was similar in both groups, but reasons differed (hypotension for barbiturates; PRIS and incomplete EEG suppression for PRO). Previous non-randomized analyses came to similar conclusions [4, 8]; while an older non-randomized study found a non-significant better RSE control and a lower mortality with PTB than PRO [19]. The primary outcome showed actually an absolute difference of $21 \%$, and a relative difference of $95 \%$; $43 \%$ of patients after PRO versus $22 \%$ after barbiturates were permanently controlled after a first course of burst-suppression. While this was not statistically significant because of the modest sample size, and the results must be viewed in the context of lack of double blinding, we believe that these data need to be addressed in future studies. It is also concerning that, overall, only $35 \%(8 / 23)$ RSE patients were treated with either agent after the first course; the fact that by the end of their hospitalization the majority of early non-responders $(9 / 15,60 \%)$ were subsequently controlled (rising the overall treatment rate to $17 / 23,73 \%$ ) may suggest that the first burst-suppression course was too short for several patients, that currently used third line SE treatments are suboptimal, or that a longer time is needed to get the underlying etiology under control. Further studies need to address this important issue.

The only significant difference between treatment groups was the longer duration of mechanical ventilation in subjects allocated to barbiturates, though without correction for multiple comparisons owing to the small sample size. PRO has a short half-life of about $140 \mathrm{~min}$ after prolonged perfusion [20], allowing a rapid titration and withdrawal. Conversely, after prolonged administration, barbiturates have a much longer elimination half-life than PRO (THP 14-36 h; PTB 15-22 h) [21, 22]. While the need of longer mechanical ventilation with barbiturates was not found in an earlier report analyzing PRO and PTB [19] and was not described in the Claassen's meta-analysis [8], it was suggested by the works of Parviainen et al. [23, 24], although these did not involve a randomized population. A significantly longer hospitalization after barbiturates as compared to PRO or midazolam was also previously described [4]. This point, implying higher costs and a greater risk of in-hospital complications, represents an important drawback for the use of barbiturates, since there is no current evidence that their efficacy on RSE might be better than with other anesthetics.

Regarding other side effects, arterial hypotension, thrombo-embolic disorders, and infections were comparable in the two arms, thus not supporting assumptions that barbiturates lead to more profound cardiovascular depression [8] or infections [25]. While those conditions may be treated relatively easily, our study confirms that coma induction in the setting of RSE may be associated with potentially fatal complications, such as gastrointestinal paralysis and PRIS. This complication was detected early and thus did not result in a fatality, underscoring the need for a serial biological monitoring in this setting (particularly lactate, $\mathrm{CK}$ and triglycerides). As compared to a recent retrospective survey reporting PRIS in 14/31 (45\%) patients receiving PRO, of whom two were fatal [26], the incidence in our prospective study is considerably lower (7\%). While this may be related to a selection bias, the concomitant use of BDZ in our study may have lowered 
this proportion. In any case, this trial confirms that RSE treatment may lead to potentially fatal complications, and should therefore carefully evaluated in those RSE groups that are felt to represent less dangerous forms (such as complex-partial RSE) $[1,7,10]$.

Finally, this trial could not be completed because of an insufficient recruitment, as after 3 years only $1 / 6$ of the target sample size was included. Several reasons accounted for this. The most important is probably related to the recruitment in this clinical setting, characterized by a "low frequency" of eligible patients (about 4-8 per year in each Center), implying a continuous and thorough information of all participants managing RSE (emergency specialists, neurologists, intensivists) about the study. Even with consequent training, the center with most included patients "missed" $45 \%$ of subjects, nearly all admitted overnight or on weekends. Moreover, the US sites could not be financially supported, as several institutions and pharmacological companies declined our applications. It is likely that lack of funding further complicated the practical management of the study in those centers, which ultimately did not join, or did not recruit any patient.

This pragmatic study confirms that RSE is a serious condition with important morbidity and mortality. Treatment of SE, and particularly RSE, mostly relies on expert opinions and data of low evidence level since several decades [6]. While it appears mandatory to improve the current situation, a future study will need to be performed by at least $25-30$ centers over several years, with a dedicated central management and adequate funding. In view of the tolerability issues related to barbiturates and PRO, inclusion of a third treatment arm (midazolam) seems advisable, even if this will increase the required sample size.

Acknowledgments The study was supported in part by grants from the Swiss League against Epilepsy, AstraZeneca (Switzerland) and UCB (Switzerland). The six authors do not have any other financial disclosures or conflict of interest. The authors wish to thank Dr Jan Novy (CHUV Lausanne), Katherine Gleason, and Sonia Replansky (BWH Boston, MA) for their help in data acquisition, and Dr Nathan B. Fountain (Charlottesville, VA) for his inputs on the study design. We confirm that we have read the Journal's position on issues involved in ethical publications and affirm that this report is consistent with those guidelines.

\section{Appendix: Clinical Trials. Gov ID: NCT00265616}

Recruiting Centers (randomized patients). Inselspital Berne, Switzerland, Drs. J. Mathis, A. Nirkko, M. Bertschi (5); Hôpitaux Universitaires de Genève, Geneva, Switzerland, Drs. P. Jallon, S. Vulliémoz, M. Seeck (2); Centre Hospitalier Universitaire Vaudois, Lausanne, Switzerland, Drs. A.O. Rossetti, J. Novy, C. Cereda, V. Rey, S. Jukopila,
M.D. Schaller (10); Brigham and Women's Hospital, Boston (MA), Drs E.B. Bromfield (deceased), J.W. Lee, T.A. Milligan (5); Massachusetts General Hospital, Boston (MA), Drs. A.J. Cole, C. Michaelides, B. Leeman (2).

\section{References}

1. Novy J, Logroscino G, Rossetti AO. Refractory status epilepticus: a prospective observational study. Epilepsia. 2010;51(2):251-6.

2. Holtkamp M, Othman J, Buchheim K, Meierkord H. Predictors and prognosis of refractory status epilepticus treated in a neurological intensive care unit. J Neurol Neurosurg Psychiatry. 2005; 76(4):534-9.

3. Mayer SA, Claassen J, Lokin J, Mendelsohn F, Dennis LJ, Fitzsimmons BF. Refractory status epilepticus: frequency, risk factors, and impact on outcome. Arch Neurol. 2002;59(2): 205-10.

4. Rossetti AO, Logroscino G, Bromfield EB. Refractory status epilepticus: effect of treatment aggressiveness on prognosis. Arch Neurol. 2005;62(11):1698-702.

5. Treiman D, Walton N, Collins J. Treatment of status epilepticus if first drug fails. Epilepsia. 1999;40(Suppl. 7):243.

6. Lowenstein DH, Alldredge BK. Status epilepticus. N Engl J Med. 1998;338(14):970-6.

7. Meierkord H, Boon P, Engelsen B, et al. EFNS guideline on the management of status epilepticus in adults. Eur J Neurol. 2010; 17(3):348-55.

8. Claassen J, Hirsch LJ, Emerson RG, Mayer SA. Treatment of refractory status epilepticus with pentobarbital, propofol, or midazolam: a systematic review. Epilepsia. 2002;43(2):146-53.

9. Schor NF, Riviello JJ Jr. Treatment with propofol: the new status quo for status epilepticus? Neurology. 2005;65(4):506-7.

10. Holtkamp M. The anaesthetic and intensive care of status epilepticus. Curr Opin Neurol. 2007;20(2):188-93.

11. Bleck TP. Refractory status epilepticus. Curr Opin Crit Care. 2005;11(2):117-20.

12. Claassen J, Hirsch LJ, Mayer SA. Treatment of status epilepticus: a survey of neurologists. J Neurol Sci. 2003;211(1-2):37-41.

13. Holtkamp M, Masuhr F, Harms L, Einhaupl KM, Meierkord H, Buchheim K. The management of refractory generalised convulsive and complex partial status epilepticus in three European countries: a survey among epileptologists and critical care neurologists. J Neurol Neurosurg Psychiatry. 2003;74(8):1095-9.

14. Rossetti AO, Logroscino G, Milligan TA, Michaelides C, Ruffieux C, Bromfield EB. Status Epilepticus Severity Score (STESS): a tool to orient early treatment strategy. J Neurol. 2008;255(10): 1561-6.

15. Rossetti AO, Reichhart MD, Schaller MD, Despland PA, Bogousslavsky J. Propofol treatment of refractory status epilepticus: a study of 31 episodes. Epilepsia. 2004;45(7):757-63.

16. Vasile B, Rasulo F, Candiani A, Latronico N. The pathophysiology of propofol infusion syndrome: a simple name for a complex syndrome. Intensive Care Med. 2003;29(9):1417-25.

17. Guidelines for epidemiologic studies on epilepsy. Commission on Epidemiology and Prognosis, International League against Epilepsy. Epilepsia 1993; 34(4):592-6.

18. Cereda C, Berger MM, Rossetti AO. Bowel ischemia: a rare complication of thiopental treatment for status epilepticus. Neurocrit Care. 2009;10(3):355-8.

19. Stecker MM, Kramer TH, Raps EC, O’Meeghan R, Dulaney E, Skaar DJ. Treatment of refractory status epilepticus with propofol: clinical and pharmacokinetic findings. Epilepsia. 1998;39(1): $18-26$. 
20. Wessen A, Persson PM, Nilsson A, Hartvig P. Clinical pharmacokinetics of propofol given as a constant-rate infusion and in combination with epidural blockade. J Clin Anesth. 1994;6(3): 193-8.

21. Bayliff CD, Schwartz ML, Hardy BG. Pharmacokinetics of highdose pentobarbital in severe head trauma. Clin Pharmacol Ther. 1985;38(4):457-61.

22. Cordato DJ, Mather LE, Gross AS, Herkes GK. Pharmacokinetics of thiopental enantiomers during and following prolonged highdose therapy. Anesthesiology. 1999;91(6):1693-702.

23. Parviainen I, Uusaro A, Kalviainen R, Kaukanen E, Mervaala E, Ruokonen E. High-dose thiopental in the treatment of refractory status epilepticus in intensive care unit. Neurology. 2002;59(8): 1249-51.

24. Parviainen I, Uusaro A, Kalviainen R, Mervaala E, Ruokonen E. Propofol in the treatment of refractory status epilepticus. Intensive Care Med. 2006;32(7):1075-9.

25. Kress HG, Segmuller R. Intravenous anesthetics and human neutrophil granulocyte motility in vitro. Anaesthesist. 1987;36(7): 356-61.

26. Iyer VN, Hoel R, Rabinstein AA. Propofol infusion syndrome in patients with refractory status epilepticus: an 11-year clinical experience. Crit Care Med. 2009;37(12):3024-30. 